\title{
Opening up to Open Notes and adding the patient to the team
}

\section{Caroline Lubick Goldzweig ${ }^{1,2}$}

'VA Greater Los Angeles Healthcare System, Los Angeles, CA

${ }^{2}$ Health Sciences Clinical Professor of Medicine, David Geffen School of Medicine at UCLA, Los Angeles, CA

\section{Correspondence to} Dr Caroline Lubick Goldzweig, 11301 Wilshire Boulevard, Mail Code 11, Los Angeles, California 90073; Caroline.Goldzweig@ va.gov

Accepted 9 June 2016 Published Online First 24 June 2016

\section{SLinked}

http://dx.doi.org/10.1136/ bmjqs-2015-004697

\section{CrossMark}

To cite: Goldzweig CL. BMJ Qual Saf 2017;26:257258.
This issue of BMJ Quality \& Safety features a paper by Bell et al ${ }^{1}$ that follows up on the original 'Open Notes' experiment with providing patients electronic access to their primary care providers' notes. In the first report, ${ }^{2}$ the intervention was well received by the patients and did not provoke the anticipated adverse impacts feared by physicians. The current paper explores the effect of Open Notes on trust within the doctor/patient relationship, again finding generally positive responses from patients and doctors. Most patients indicated that they accessed notes to better understand and learn more about their health; and reading notes either did not change their feelings or made them feel better about their doctors. Most physicians thought that patient satisfaction had improved.

The concept of Open Notes builds upon the increasingly prevalent practice of giving patients electronic access to at least some components of their medical records through the use of patient portals tethered to provider electronic health records (EHRs). These portals are a natural extension of the growth in EHR use across the USA driven in large part by the HITECH Act and the Center for Medicare and Medicaid's 'meaningful use' programme. But they also reflect our increasingly electronic lifestyle and our reliance on the internet for information, connection and communication.

Because patient portals are a new technology, their full impact in healthcare has likely not been realised, and having access to health information through a portal does not necessarily guarantee better healthcare outcomes or increased efficiency. While the literature around these portals continues to grow, results for various endpoints are mixed. ${ }^{3-6}$ Some studies report improvements in patient satisfaction, but many demonstrate no change. Impacts on clinical outcomes like improved blood pressure or diabetes control are also variable and sometimes dependent on whether the intervention is coupled with case management. Studies of efficiencies may demonstrate increased documentation time for physicians and none have demonstrated time-savings. Studies related to patient empowerment, including the study at hand, while not universally positive, do identify benefits from the patient perspective. ${ }^{7}$ So while it may be difficult to elicit definitive disease-based outcome benefits from patient portals, the real question at hand may be how these healthcare tools can be optimised to improve shared decisionmaking and team-based, patient-centred care.

In many respects, patient portals, and Open Notes in particular, could be key elements in facilitating shared decisionmaking, which has been associated with improved patient outcomes. ${ }^{8}$ Studies have identified a number of factors that improve patient engagement in healthcare decisions including skills and knowledge, patient/provider relationships built on trust, good communication mechanisms and decreased confusion about choices. $^{9} 10$ This study of Open Notes, similar to others, ${ }^{7} 11$ has shown that patients believe having access to notes improves their knowledge and communication with their providers and, notably, that easy access to their notes can improve trust with their providers. From this perspective, Open Notes would seem like an obvious adjunct to efforts to enhance shared decision-making.

Healthcare delivery is increasingly team-based where the physician is no longer functioning as the 'director', but is a collaborator with the patient, serving as a subject matter expert, and working with other team members to ensure the best care and experience for the patient. Patient access to information and education is one of eight dimensions of patient-centred care. ${ }^{12}$ When patients understand their clinical status, their prognosis and critical processes involved 
in their care, whether from discussions with their providers or by reading their progress notes, they have a greater sense of autonomy and are better able to engage in self-care.

Just as doctors have had to adjust over time to new technologies, new paradigms regarding disease and new approaches to the science of medicine, they are adapting to a team-based delivery model that includes the patient and facilitates more open access to their thought processes and management rationales. The Open Notes experiment shows that patients want to be on the same team as their doctors, want to understand what the doctor thinks and want to follow doctors' 'orders'. And having easy access to their notes has not resulted in significant negative repercussions, at least in the case of access to primary care notes. Given this, and the potential for positively impacting on shared decision-making and team-based, patient-centred care, Open Notes is an obvious intervention for systems with EHRs and patient portals. This does not mean that it will be business as usual, and healthcare providers will need to modify their documentation practices to ensure clarity, objectivity and comprehensiveness. This may be particularly important if mental health notes become more widely available, as they have through the US Department of Veterans Affairs. This is one area that has not been specifically studied, and where there may be unique risks and stronger physician pushback than for primary care or other specialty visit notes.

Perhaps the biggest barrier to realising the potential benefits of patient portals and Open Notes is finding ways to ensure that all patients can access them. Previous studies have identified a sociodemographic divide in who uses these portals with those who are poorer, less educated and from racial and ethnic minorities being less likely to participate. ${ }^{6}$ A recent news article highlighted the increasing challenges to success for those who have no reliable internet access. ${ }^{13}$ The Open Notes participants were significantly more likely to be college educated and white, though this group was not the only one to benefit, with analyses of non-white and poorer participants demonstrating similar positive results. Improving internet access and computer literacy must be a public health priority, to ensure we combat yet another potential reason for variations in healthcare access and quality based on race or income.

The lack of evidence for harm from open access to physician notes, combined with our society's reliance on information technology to navigate the world and inform decisions, makes Open Notes an inevitable part of medical care delivery. It is clear that its use will continue to spread as more and more healthcare organisations expand their patient portals and adopt this practice. The medical community should embrace Open Notes while focusing on identifying best practices to enhance the patient experience and expand the potential for shared decision-making.

Competing interests None declared.

Provenance and peer review Commissioned; internally peer reviewed.

\section{REFERENCES}

1 Bell SK, Mejilla R, Anselmo M, et al. When doctors share visit notes with patients: a study of patient and doctor perceptions of documentation errors, safety opportunities, and the patient-doctor relationship. BMJ Qual Saf 2017;26:262-70.

2 Delbanco T, Walker J, Bell SK, et al. Inviting patients to read their doctors' notes: a quasi-experimental study and a look ahead. Ann Intern Med 2012;157:461-70.

3 Jilka SR, Callahan R, Sevdalis N, et al. "Nothing about me without me": an interpretative review of patient accessible electronic health records. J Med Internet Res 2015;17:e161.

4 Kruse CS, Bolton K, Freriks G. The effect of patient portals on quality outcomes and its implications to meaningful use: a systematic review. J Med Internet Res 2015;17:e44.

5 Ammenwerth E, Schnell-Inderst P, Hoerbst A. The impact of electronic patient portals on patient care: a systematic review of controlled trials. J Med Internet Res 2012;14:e162.

6 Goldzweig CL, Orshansky G, Paige NM, et al. Electronic patient portals: evidence on health outcomes, satisfaction, efficiency, and attitudes. A systematic review. Ann Intern Med 2013;159:677-87.

7 Nazi KM, Turvey CL, Klein DM, et al. VA Open Notes: exploring the experiences of early patient adopters with access to clinical notes. J Am Med Inform Assoc 2015;22:380-9.

8 Shay LA, Lafata JE. Where is the evidence? A systematic review of shared decision making and patient outcomes. Med Decis Making 2015;35:114-31.

9 Elliott J, McNeil H, Ashbourne J, et al. Engaging older adults in health care decision-making: a realist synthesis. Patient 2016.

10 Fraenkel L, McGraw S. What are the essential elements to enable patient participation in medical decision-making? J Gen Intern Med 2007;22:614-9.

11 Woods SS, Schwartz E, Tuepker A, et al. Patient experiences with full electronic access to health records and clinical notes through the My HealtheVet personal health record pilot: qualitative study. J Med Internet Res 2013;15:e65.

12 Eight Dimensions of PCC. http://www.nationalresearch.com/ products-and-solutions/patient-and-family-experience/ eight-dimensions-of-patient-centered-care/ (accessed 2 Jun 2016).

13 Kang C. Unemployed Detroit residents are trapped by a digital divide. New York Times 22 May 2016. http://www.nytimes. com/2016/05/23/technology/unemployed-detroit-residentsare-trapped-by-a-digital-divide.html (accessed 2 Jun 2016). 